\title{
¿Evalúo cómo me Evaluaron en la Facultad? Transferencia de la Evaluación Formativa y Compartida Vivida durante la Formación Inicial del Profesorado a la Práctica como Docente
}

\author{
Do I Evaluate as I was at the Faculty? Transfer of the Formative \\ and Shared Evaluation Lived during the Initial Teacher \\ Training to Teaching Practice
}

\author{
Miriam Molina * \\ Víctor M. López-Pastor \\ Universidad de Valladolid, España
}

\begin{abstract}
La finalidad de este estudio es analizar la trasferencia entre los sistemas de evaluación formativa $\mathrm{y} / \mathrm{o}$ compartida $(\mathrm{EFyC})$ que el profesorado de educación física puede haber vivido durante su formación inicial del profesorado (FIP) y su aplicación posterior en las aulas de educación primaria en que ejerce su docencia actualmente. Se ha aplicado una metodología mixta, con una muestra reducida de maestros de educación física de primaria. Las técnicas de obtención de datos son: cuestionario cerrado con escala tipo Likert, entrevistas semi-estructuradas y un grupo focal. En primer lugar, se aplica el cuestionario con toda la muestra, de forma que los resultados obtenidos nos ayuden a seleccionar una pequeña muestra para la realización de las entrevistas semiestructuradas. Los datos cuantitativos se analizan con el programa SPSS 20.0 y los cualitativos con el programa informático Atlas.ti. Los resultados indican que sí existe cierta trasferencia entre los sistemas de evaluación vividos en FIP y los que posteriormente se utilizan en la práctica como maestros de educación física en primaria; pero parece que la influencia más fuerte es de la formación permanente, por lo que parece necesario realizar más investigación al respecto. Los maestros de educación física de este estudio están convencidos de las ventajas que supone la utilización de sistemas de $\mathrm{EFyC}$ en todos los ámbitos educativos, tanto para el alumnado como para el profesorado.
\end{abstract}

Palabras clave: Evaluación formativa; Evaluación compartida; Formación del profesorado; Educación física, Cambio educativo.

This study analyses to which extent formative and shared assessment experiences received during their Physical Education Teacher Education (PETE) can influence to put into practice this type of systems in Physical Education teachers in Primary Education. A mixed methodology has been used, with a reduced sample. The data collection techniques are: closed questionnaire with Likert-type scale, semi-structured interviews and a focus group. In the first place, the questionnaire is applied to the whole sample, so that the results obtained help us to select a small sample for semi-structured interviews. The quantitative data are analyzed with the SPSS 20.0 program and the qualitative ones with the Atlas.ti. The results to indicate that there is some transference between the assessment systems lived in PTE and those that are later used later when they are Physical Education teachers in Primary; but it seems that the strongest influence is In-service Teacher Education, so it seems necessary to conduct more research in this regard. The physical education teachers of this study are convinced of the advantages of using formative and shared assessment systems in all educational areas, both for students and teachers.

Keywords: Formative assessment; Shared assessment; Teacher education; Physical education, Educational change.

*Contacto: miriam.molina@uva.es

issn: 1989-0397

www.rinace.net/riee/

https://revistas.uam.es/riee
Recibido: $\quad 15$ de enero de 2019

$1^{\text {a }}$ Evaluación: 04 de febrero de 2019

$2^{\text {a }}$ Evaluación: 01 de marzo d 2019

Aceptado: $\quad 12$ de marzo de 2019 


\section{Introducción}

Este artículo se centra en la temática de la Evaluación Formativa y Compartida (EFyC) en la formación inicial del profesorado (FIP), así como su posible influencia posterior en los docentes en su práctica diaria. Para ello, se organiza la revisión de la literatura en tres grandes apartados: (1) la EFyC en Educación Física; (2) la importancia de las experiencias de EFyC en FIP y en la FIP de Educación Física; y, (3) la transferencia entre lo vivido en la FIP y la futura práctica en primaria. Posteriormente se presenta la metodología utilizada en el estudio, los resultados, la discusión y las conclusiones.

\section{Revisión de la literatura}

\subsection{La Evaluación Formativa y Compartida en Educación Física}

Durante las últimas décadas se busca el cambio de una evaluación centrada en la calificación, a una evaluación centrada en el aprendizaje (formativa y continua). Según Castejón, Santos y Palacios (2015) "la evaluación debe vincularse con el concepto de evaluación formativa, así podremos comprobar los resultados del proceso de aprender y no sólo los resultados que tratan la evaluación como sinónimo de calificación” (p.247).

En López-Pastor y Pérez-Pueyo (2017) encontramos una definición actual de evaluación formativa: "hace referencia a todo proceso de evaluación cuya finalidad principal es mejorar los procesos de enseñanza-aprendizaje que tienen lugar” (p.36). Asimismo, LópezPastor (2009) considera que la EFyC es: "todo proceso de constatación, valoración y toma de decisiones cuya finalidad es optimizar el proceso de enseñanza-aprendizaje que tiene lugar, desde una perspectiva humanizadora y no como mero fin calificador" (p.35).

En el cuadro 1 presentamos las ventajas e inconvenientes que presentan los sistemas de EFyC en la FIP, según la opinión de los estudiantes universitarios.

Según Hamodi y otros (2017), los "maestros noveles" que recibieron EFyC en su FIP aseguran haber logrado un aprendizaje más profundo. Los "maestros noveles" son maestros incorporados recientemente a su práctica docente en las aulas. Barrientos, López-Pastor y Molina (2018) realizan un estudio con maestros de educación física en primaria y secundaria en el que se resumen tres ventajas principales de utilizar sistemas de EFyC en sus aulas: (1) los estudiantes son más conscientes de la evaluación y de los objetivos que deben alcanzar durante su proceso, (2) la participación de los estudiantes en su evaluación ayuda a su aprendizaje, y (3) la retroalimentación o feedback regular ayuda a los estudiantes a mejorar su proceso de aprendizaje.

López-Pastor y Pérez-Pueyo (2017) realizan una recopilación de buenas prácticas en $\mathrm{EF} y \mathrm{C}$ en todas las etapas educativas, desde educación infantil hasta universidad, en la que pueden comprobarse las ventajas de este modelo. Los resultados encontrados en todas estas experiencias parecen apuntar algunas conclusiones: (a) en muchos casos se necesita al menos un trimestre de funcionamiento para que estos sistemas tengan resultados positivos; (b) el proceso de EFyC ayuda a la mejora de los aprendizajes de los alumnos; y (c) en todas las experiencias se han obtenido resultados positivos.

También debemos tener en cuenta los inconvenientes que este tipo de sistemas de evaluación presentan en la práctica real en las aulas; la principal desventaja es la carga de 
trabajo. Mintah (2003) realizó un estudio en el que el 75\% de la muestra de los maestros pusieron en práctica evaluación auténtica (véase cuadro 2) en sus aulas de educación primaria, pero el resto de la muestra de los maestros consideraban que estos sistemas tenían una carga trabajo excesiva y su formación en los ámbitos de evaluación no era suficiente. Esto coincide con otros estudios, que aseguran que los sistemas de $\mathrm{EFyC}$ tienen una excesiva carga de trabajo, tanto para el alumnado como para el profesorado (MartínezMínguez et al., 2015; Hamodi et al., 2017; López-Pastor y Pérez-Pueyo, 2017), aunque también aseguran que funcionan mejor si se hace partícipes a los alumnos en su proceso de aprendizaje, ya que incrementará su interés y motivación, además de mejorar la calidad del aprendizaje. En el estudio de Ibarra, Rodríguez y Gómez (2012), los profesores y el alumnado también plantean resistencias al utilizar evaluación compartida (véase tabla 2) por el cambio metodológico que exige, ya que este tipo de evaluación supone un mayor esfuerzo por parte de profesorado y alumnado.

Cuadro 1. Ventajas e inconvenientes de la EFyC en la FIP

\begin{tabular}{l}
\hline \multicolumn{1}{c}{ VENTAJAS } \\
\hline Mejora la motivación e implicación del alumnado en el proceso de aprendizaje. \\
Ayuda a corregir a tiempo los problemas del proceso de enseñanza-aprendizaje. \\
Es una experiencia de aprendizaje en sí misma. Existe una retroalimentación y posibilidad de \\
corregir y aprender de los errores. \\
Facilita el desarrollo de competencias personales y profesionales. \\
Desarrolla la autonomía y la responsabilidad del alumnado. Requiere más responsabilidad. \\
Mejora el rendimiento académico, gracias al aprendizaje activo del alumnado. \\
Ofrece alternativas a todos los estudiantes. \\
Existe un acuerdo previo del sistema de evaluación. \\
Se centra en el proceso y no en el trabajo final. \\
La calificación es más justa, gracias al seguimiento más individualizado. \\
Mejora la calidad de los trabajos exigidos. \\
El alumnado aprende más y mejor
\end{tabular}

El alumnado aprende más y mejor

Exige continuidad: asistencia obligatoria y activa.

La dinámica de trabajo es poco conocida y existe falta de hábito.

Requiere un mayor esfuerzo.

Se presentan dificultades para trabajar en grupo.

Puede que el proceso de calificación sea poco claro y se genere inseguridad durante el proceso.

La valoración de los trabajos es subjetiva.

Exige una autoevaluación de mi propio proceso.

Se puede acumular mucho trabajo al final del proceso.

Existe una desproporción entre el tiempo de trabajo y los créditos de la asignatura.

Las correcciones pueden ser poco claras.

Fuente: Elaboración propia basado en López-Pastor (2012), Martínez-Mínguez, Vallés y RomeroMartín (2015) y Hamodi, López-Pastor y López-Pastor (2017).

López-Pastor y Pérez-Pueyo (2017) defienden que la implicación del alumnado en éstos sistemas de evaluación es imprescindible para su buen funcionamiento porque: (a) mejora de sus aprendizajes; (b) mejora su autonomía personal, autorregulación y proceso de aprender a aprender; (c) desarrolla la capacidad de análisis crítico; (d) ayuda a formar personas responsables; y (e) mejora del clima de aula y resolución de problemas de convivencia. Según López-Pastor (2012, 2013) e Ibarra et al. (2012) esta implicación se puede dar a través de coevaluación (o evaluación entre iguales), autoevaluación, evaluación compartida y calificación dialogada (véase cuadro 2). 
Cuadro 2. Aclaración de conceptos sobre la participación del alumnado en evaluación

\begin{tabular}{|c|c|}
\hline AUTOEVALUACIÓN & $\begin{array}{l}\text { Evaluación que realiza una persona sobre sí misma, sobre su proceso de } \\
\text { enseñanza-aprendizaje. Puede ser individual o grupal, tanto de alumnado } \\
\text { como de profesorado. }\end{array}$ \\
\hline COEVAluación & Es la evaluación entre iguales, y puede ser individual o grupal. \\
\hline $\begin{array}{l}\text { EVALUACIÓN } \\
\text { COMPARTIDA }\end{array}$ & $\begin{array}{l}\text { El profesor dialoga con el alumnado sobre la evaluación de sus } \\
\text { aprendizajes y del proceso de enseñanza-aprendizaje. Este diálogo puede } \\
\text { ser a nivel individual o grupal. }\end{array}$ \\
\hline $\begin{array}{l}\text { CALIFICACIÓN } \\
\text { DIALOGADA }\end{array}$ & $\begin{array}{l}\text { Es un proceso en el que el alumnado y el profesorado dialogan sobre la } \\
\text { calificación, tomando como base los criterios de calificación que están } \\
\text { previamente establecidos. Este diálogo se puede realizar a nivel } \\
\text { individual, en pequeños grupos o en gran grupo. }\end{array}$ \\
\hline $\begin{array}{l}\text { EVALUACIÓN } \\
\text { AUTÉNTICA }\end{array}$ & $\begin{array}{l}\text { Las técnicas, los instrumentos y las actividades de evaluación deben estar } \\
\text { implicados en contenidos reales de aprendizaje o relacionados con } \\
\text { competencias profesionales. }\end{array}$ \\
\hline
\end{tabular}

Fuente: Elaboración propia a partir de López-Pastor (2012, 2013).

\subsection{La importancia de las experiencias de EFyC en FIP y en la FIP de Educación Física}

En la literatura especializada pueden encontrarse numerosos trabajos sobre experiencias y estudios de la aplicación de la EFyC en la FIP de educación física. El estudio de Palacios y López (2013) tiene como muestra 80 docentes de las Facultades de Ciencias de la Actividad Física y el Deporte y las Escuelas de Magisterio encargados de dicha FIP. Los resultados encontrados muestran que existen tres tipos de profesorado en FIP de educación física: "innovador" (con actitud positiva hacia sistemas de $\mathrm{EFyC}$ ), "tradicional" (el alumnado no participa en la evaluación y la calificación sale de forma total o principal del examen final) y "ecléctico" (muestra una actitud positiva hacía la utilización de sistemas de $\mathrm{EFyC}$, pero sigue teniendo gran protagonismo el examen final). Los resultados muestran que el $49 \%$ de los profesores son eclécticos, el $26 \%$ son tradicionales y el $25 \%$ son innovadores. Son datos muy positivos si se comparan con otros estudios más antiguos (Fernández, 1989; Porto, 1998; Tejedor, 1998; Flórez, 1999; Santos, 1999; Trillo, 1999), en los que predominaba el modelo de evaluación "tradicional", basado en el examen final, lo que significa que en la FIP se están produciendo cambios en los sistemas de evaluación.

Hay varias razones por las que es importante desarrollar sistemas de EFyC en FIP y, concretamente, en la FIP de educación física:

\section{a) Mejora el aprendizaje del alumnado}

Los estudios realizados sobre el desarrollo de sistemas de EFyC en FIP muestran que el alumnado valora positivamente el mayor grado de aprendizaje que suelen generar (Martínez-Mínguez et al., 2015; Hamodi, et al. 2017; López-Pastor y Pérez-Pueyo, 2017). Estos resultados coinciden con los planteamientos de Dochy et al. (2002), que defendía que las universidades europeas deberían asumir el reto de ir cambiando sus sistemas de evaluación, de modo que se pase de la "cultura del examen" (evaluación tradicional) a la "cultura de la evaluación", más centrada en los procesos de aprendizaje del alumnado.

Hamodi y otros (2017) encuentran que los maestros consideran que la utilización de sistemas de EFyC durante su FIP les ayudó a lograr un aprendizaje más profundo; por tanto, sería lógico que el alumnado de FIP experimente sistemas de evaluación diferentes a los tradicionales, de modo que promuevan un mayor aprendizaje. Parece existir una gran 
influencia de la evaluación recibida durante la FIP en la evaluación que aplican posteriormente los maestros de educación física en su práctica docente en las aulas de primaria. Esto es, la experiencia de haber vivido sistemas de EFyC durante su FIP parece que ayuda al profesorado novel a aplicar sistemas de $\mathrm{EFyC}$ en su práctica actual como maestros, a pesar de haber encontrado barreras para introducir métodos evaluativos innovadores en educación primaria. (Hamodi et al., 2017; Molina y López-Pastor, 2017).

\section{b) Ayuda a mejorar el rendimiento académico del alumnado}

Algunos estudios (Fraile et al., 2013; López-Pastor, 2009; Romero et al., 2014; Santos, Martínez y López-Pastor, 2009) comprueban que la utilización de sistemas de EFyC genera un mejor rendimiento académico que los sistemas tradicionales centrados en un examen final. Consideran que se trata de un efecto lógico, dado que se mejora el proceso de enseñanza-aprendizaje, la implicación del alumnado en su propio aprendizaje y la capacidad de aprender a aprender. Los mismos resultados se han encontrado en todas las etapas educativas (López-Pastor y Pérez-Pueyo, 2017).

En este sentido, el estudio realizado por Buscà et al. (2010), con una muestra elevada y diversa (40 profesores y 2491 alumnos de FIP de toda España), encuentra que los estudiantes de FIP suelen participar en coevaluación y autoevaluación de algunas actividades de aprendizaje, pero que el profesorado no cede la responsabilidad al alumnado para llegar a la calificación final, salvo unos pocos profesores, que sí realizan procesos de calificación dialogada. Por ello, estos autores consideran que la participación del alumnado en su evaluación genera unos resultados académicos más satisfactorios.

\section{c) Ayuda a desarrollar la competencia docente de la evaluación educativa}

Diferentes autores consideran que la EFyC durante la FIP ayuda a desarrollar la competencia evaluativa, que van a necesitar dominar en un futuro próximo, cuando los hoy alumnos ejerzan como docentes (Hamodi et al., 2017; López-Pastor, 2008, 2013; Lorente y Kirk, 2013; Manrique et al., 2011; Palacios y López-Pastor, 2013; RamírezGarcía, González-Fernández y Salcines-Talledo, 2018). La hipótesis que manejan los autores es que, si los maestros no han experimentado durante su FIP sistemas de EFyC, es difícil que los utilicen cuando sean maestros, por lo que no ayudarán a mejorar los aprendizajes de su futuro alumnado y a favorecer su implicación en los procesos de la evaluación.

Por ello, los modelos de EFyC en la FIP parecen ser la evaluación más lógica cuando se está realizando sistemas centrados en el aprendizaje del alumnado y el desarrollo de competencias, tanto personales como profesionales (López-Pastor, 2008, 2009, 2012; Fraile et al., 2013; Hamodi, 2014; López-Pastor y Pérez-Pueyo, 2017; Lorente, LópezPastor y Kirk, 2018).

\section{d) Suele generar una elevada satisfacción en el alumnado y el profesorado}

El alumnado muestra gran satisfacción con la utilización de sistemas de EFyC durante su FIP porque mejora su aprendizaje y su rendimiento académico (Castejón et al., 2011; López-Pastor, 2012; López-Pastor y Pérez-Pueyo, 2017; Romero, Castejón y LópezPastor; 2015; Silva y López-Pastor, 2015), aunque considera que este tipo de sistemas genera mucha carga de trabajo y compromiso (Castejón et al., 2011). Cañadas (2018) considera que las razones por las que el alumnado se muestra satisfecho son: (a) constante 
feedback por parte del profesorado; (b) implicación en el propio proceso de aprendizaje y en el de los compañeros; (c) desarrollo de debates que pongan en evidencia el aprendizaje del alumnado; y (d) mejora de la responsabilidad, autonomía y la autorregulación del alumnado.

El profesorado también suele estar más satisfecho con la implicación y los resultados de su alumnado cuando se utilizan este tipo de sistemas de evaluación (López-Pastor, 2009; Romero et al, 2015).

\subsection{La transferencia entre lo vivido en la Formación Inicial del Profesorado y la futura práctica en primaria}

Parecen existir pocos estudios sobre la transferencia entre la evaluación vivida en la FIP y la posterior aplicación en el aula. En este apartado realizaremos una pequeña revisión sobre algunos de los resultados encontrados. Por ejemplo, Lorente y Kirk (2013) aseguran que es muy importante que los docentes vivan y experimenten sistemas de $\mathrm{EFyC}$ durante su FIP para que sean competentes en dichos métodos de evaluación en las escuelas actuales. Hamodi et al. (2017) encuentran que parece existir una gran influencia de la evaluación recibida durante la FIP en la evaluación que aplican posteriormente los maestros de educación física en su práctica docente en las aulas de primaria. Esto es, la experiencia de haber vivido sistemas de EFyC durante su FIP ha ayudado al profesorado novel a aplicar sistemas de EFyC en su práctica actual como maestros, a pesar de haber encontrado barreras para introducir métodos evaluativos innovadores en educación primaria.

En el último año se han publicado diferentes estudios sobre la influencia que pueden tener los sistemas de evaluación vividos durante la FIP y su transferencia posterior a la práctica real en las aulas de educación obligatoria. Por ejemplo, los resultados encontrados por Molina, López-Pastor y Barrientos (2018) y Molina y López-Pastor (2017), indican que sí existe esa trasferencia. Los maestros de educación física entrevistados consideran imprescindible la experimentación de sistemas de EFyC durante su FIP para su posterior aplicación en las aulas, en cualquier nivel educativo y de cualquier materia. Sin embargo, Barrientos et al. (2018) encuentran otros resultados, cuando entrevistan a profesores de educación física que no tuvieron experiencias de EFyC durante su FIP, ni recibieron formación sobre este tipo de evaluación. Descubren que los profesores de educación física de primaria se implican en procesos de formación permanente del profesorado (FPP) que les ayuda a aprender y avanzar en la aplicación de sistemas de EFyC en su aula cuando se dan dos condiciones: (a) se hacen conscientes de esa falta de formación, durante sus primeros años como docentes; $\mathrm{y}$ (b) tienen claro que no quieren seguir reproduciendo los sistemas tradicionales de evaluación en educación física. En estos procesos de desarrollo profesional, las vías de FPP que parecen haber influido más son: integrarse en grupos de trabajo con maestros que utilizan sistemas de $\mathrm{EFyC}$, asistencia a cursos sobre la temática, asistencia a congresos donde conocen la propuesta y/o leer bibliografía relacionada con este tipo de evaluación.

En definitiva, son muchos los autores que consideran que los sistemas de EFyC deberían estar presentes en la FIP si queremos que posteriormente el profesorado lo trasfiera a su aula (Hamodi et al., 2017; Lorente-Catalán y Kirk, 2016; Lorente et al., 2018; Palacios y López-Pastor, 2013). Además, parece conveniente que formen parte de actividades de FPP (seminarios, grupos de trabajo, proyectos en centro), donde poder trabajar de forma 
colaborativa con otros docentes que también utilizan sistemas de $\mathrm{EFyC}$, para ir realizando mejoras progresivas en la aplicación a contextos educativos concretos (Barrientos et al., 2018; Barrientos, López-Pastor y Pérez-Brunicardi, 2019; Hamodi et al., 2017).

La transferencia de la EFyC en la FIP de EF es un tema poco investigado y del que existen pocas propuestas prácticas. Por ello, los objetivos que persigue esta investigación son:

1. Examinar en qué medida la EFyC experimentada durante la FIP ha influido en el tipo de evaluación que realizan actualmente en sus aulas los maestros de educación física de primaria.

2. Explorar que otros aspectos de la formación inicial o permanente han influido para que el profesorado de educación física de primaria utilice sistemas de EFyC en sus aulas.

\section{Método}

\subsection{Enfoque metodológico}

El diseño de esta investigación es un estudio de caso en el que se analiza una muestra de maestros de educación física que utilizan sistemas de $\mathrm{EFyC}$ en educación primaria. El estudio de caso es "el estudio de la particularidad y de la complejidad de un caso singular, para llegar a comprender su actividad en circunstancias importantes" (Stake, 2005, p.11). Se trata de estudiar un fenómeno concreto en un contexto real de nuestra sociedad.

\subsection{Participantes}

En un primer momento se realizaron tres grandes preguntas a una muestra intencionada de 22 maestros de educación física que sabíamos que utilizaban $\mathrm{EFyC}$ en sus aulas, en mayor o menor medida, y que la mayoría de ellos había vivido este tipo de sistemas de evaluación en algunas asignaturas durante su FIP. Se les realizaron tres preguntas, enfocadas a avanzar en los objetivos de investigación. Son las siguientes:

- ¿Dónde han recibido su FIP?

- ¿Trabajan como profesores especialistas de educación física en primaria?

- ¿Utilizan habitualmente sistemas de EFyC en sus clases?

Tras las respuestas recogidas, la muestra definitiva es de 17 maestros de educación física de educación primaria, de una misma provincia, que son los que utilizaban $\mathrm{EFyC}$ en sus aulas, que realizaron el cuestionario cerrados con escala tipo Likert.

Por otra parte, el grupo focal está formado por 16 egresados, pertenecientes a tres Facultades de Educación españolas de tres comunidades autónomas diferentes. Su edad media es de 27, con un margen de +/- 2-3 años, según el caso. En todos los casos se trata de egresados que estaban trabajando en ese momento como profesores de educación física y que habían experimentado sistemas de EFyC durante su FIP.

\subsection{Instrumentos de obtención de información:}

En esta investigación se combinan instrumentos cuantitativos (cuestionarios cerrados con escala tipo Likert) e instrumentos cualitativos (entrevistas semiestructuradas y un grupo focal). 
El cuestionario consta de seis ítems en los que se calcula la media aritmética con una escala 1-4 (escala: 1, nada de acuerdo; 2, poco de acuerdo; 3, bastante de acuerdo y, 4, muy de acuerdo) y la desviación típica (DT). El cuestionario se divide en dos bloques:

- Bloque 1: ítems sobre los sistemas de evaluación vividos en su FIP.

- Bloque 2: ítems sobre los sistemas de evaluación y calificación que utilizan con el alumnado.

El cuestionario cerrado con escala tipo Likert fue diseñado por los autores del trabajo y validado por tres investigadores especializados en la temática. La validación se realizó por separado y por vía electrónica, anotando los cambios necesarios en el cuestionario. Las recomendaciones y erratas fueron utilizadas para elaborar la versión definitiva.

Las entrevistas son semiestructuradas y las preguntas son:

1. ¿Por qué comienzas a utilizar la EFyC en tus clases de educación física?

2. ¿Qué formación tienes sobre este tipo de evaluación?

3. ¿Tuviste nuevas experiencias de evaluación formativa o de EFyC durante tu FIP?

4. En caso positivo: ¿Cómo y cuánto crees que ha influido esa experiencia a la hora de que ahora apliques sistemas o procesos de evaluación formativa en tus clases?

5. ¿Qué importancia crees que tiene el uso de la EFyC con tus alumnos?

Este grupo focal se realizó en una universidad de Madrid, por ser el punto intermedio de las tres universidades implicadas. El grupo consta de 16 personas, egresados recientes de tres facultades que han experimentado sistemas de $\mathrm{EFyC}$ durante su FIP. El grupo focal se grabó en vídeo y audio y se realizó una trascripción completa del mismo. El tema principal del grupo focal es "Las competencias docentes en la formación inicial del profesorado de EF", aunque en nuestro estudio nos centraremos en los resultados obtenidos sólo sobre la evaluación.

\subsection{Trabajo de campo}

En primer lugar se aplica el cuestionario a la muestra seleccionada de 17 maestros de educación física de primaria, entre los cuales se eligen tres casos diferentes entre sí respecto a la FIP recibida en sistemas de EFyC. Posteriormente se realizó la entrevista semiestructurada con los tres maestros seleccionados. En tercer lugar, se realizó el grupo focal de egresados que han experimentado sistemas de EFyC durante la FIP.

\subsection{Categorías de análisis}

Tanto los resultados como la discusión se organizan a partir de los dos objetivos planteados en este estudio (véase cuadro 3).

Cuadro 3. Categorías de análisis

\begin{tabular}{ll}
\hline \multicolumn{1}{c}{ OBJETIVOS } & \multicolumn{1}{c}{ CATEGORÍAS } \\
\hline Examinar en qué medida la EFyC experimentada durante la FIP & Influencia de la FIP \\
ha influido en el tipo de evaluación que realizan actualmente en & Formación en EFyC \\
sus aulas los maestros de educación física de primaria. & Calificación \\
Explorar que otros aspectos de la formación inicial o permanente & $\begin{array}{l}\text { Participación del alumnado } \\
\text { han influido para que el profesorado de educación física de } \\
\text { primaria utilice sistemas de EFyC en sus aulas. }\end{array}$ \\
& $\begin{array}{l}\text { en evaluación } \\
\text { Aplicabilidad de EFyC }\end{array}$ \\
\hline
\end{tabular}

Fuente: Elaboración propia. 


\subsection{Análisis de datos}

$\mathrm{El}$ análisis de datos se realiza a través de dos programas: (a) SPSS 20.0 para los datos del cuestionario; y (b) Atlas.ti 7.3 para los datos recogidos con las entrevistas semiestructuradas y el grupo focal.

Los resultados del cuestionario se recogen en tablas con la media aritmética y DT, calculadas con el programa SPSS 20.0. La media puede tener un máximo de 4 puntos.

La transcripción de las entrevistas se realiza con un código que identificará a las personas entrevistadas, pero manteniendo el anonimato. El código irá entre paréntesis, al final de cada cita literal. Por ejemplo, el código: "ES-3,5" indica: entrevista semiestructurada, maestro 3. El grupo focal seguirá el mismo código. Por ejemplo, el código: "GF-2" nos indica: intervención del participante 2 en el grupo focal.

\section{Resultados}

Los resultados se analizarán triangulando datos de los cuestionarios, entrevistas y grupo focal con los objetivos marcados al inicio de esta investigación.

\subsection{Objetivo 1: examinar en qué medida la EFyC experimentada durante la FIP ha influido en el tipo de evaluación que realizan actualmente en sus aulas los maestros de educación física de primaria}

En la tabla 3 podemos comprobar cómo los maestros de educación física encuestados aportan valores medios en el ítem que pregunta si durante la FIP han vivido sistemas de EFyC en algunas asignaturas $(2,44)$ y con que los sistemas de EFyC vividos en la FIP han influido en los sistemas de evaluación que ahora utilizan como maestros $(3,39)$.

Cuadro 4. Resultados de los ítems sobre los sistemas de evaluación vividos por maestros de educación física en su FIP (escala Likert 1-4)

\begin{tabular}{lcc}
\hline \multicolumn{1}{c}{ ÍTEMS. GRADO DE ACUERDO CON } & MEDIA & DT \\
\hline $\begin{array}{l}\text { Durante mi FIP he vivido sistemas de evaluación formativa y/o } \\
\text { compartida en algunas asignaturas }\end{array}$ & 2,44 & 1,097 \\
$\begin{array}{l}\text { Los sistemas de evaluación formativa vividos en mi FIP han influido en } \\
\text { los sistemas de evaluación que ahora utilizo como maestro. }\end{array}$ & 2,39 & 1,243 \\
\hline
\end{tabular}

Fuente: Elaboración propia.

Las entrevistas realizadas permiten profundizar en estos resultados. Mientras que unos maestros hacen referencia a que durante la FIP sí han recibido sistemas de EFyC y después han seguido formándose en el tema; otros, no experimentaron durante su formación este tipo de sistemas de evaluación, lo que ha provocado una formación autónoma.

\footnotetext{
Mi formación en evaluación formativa es lo que me hizo continuar investigando y cogiendo información sobre cómo podía ser la evaluación formativa. (ES-2,5)

Cuando yo estudié magisterio apenas vimos nada de todo esto; nos dieron algunas pinceladas y algunas pequeñas ideas. Ha sido más autoformación que lo que yo he visto en la universidad. (ES-1,5)
}

Uno de los egresados del grupo focal trabaja actualmente como profesor universitario, en la FIP de educación física, y considera que se ha producido un cambio en la FIP de las universidades españolas, pero aún queda mucho trabajo por hacer, ya que hay un enorme 
desconocimiento sobre este tipo de sistemas; otros maestros que trabajan en primaria aportan más comentarios en este mismo sentido:

Todos mis amigos están haciendo evaluación formativa, entonces, mis amigos profesionales estamos en este rollo. Entonces, mi percepción es que la perspectiva no está tan mal como se piensa porque todos los que tengo a mi alrededor hacen innovación. Pero si abro un poquito el campo y miro los que hay, no ya de los de mi departamento si no los de al lado, es que... no hay nada. (GF-3)

Lo que había era mera educación bancaria. Era dictar apuntes, memorizarlo, repetirlo en un examen y olvidarlo. $Y$ esa es la universidad en la que hemos crecido todos. Claro, eso es lo que teníamos [...] Frente a esos modelos las competencias lo que nos ofrece, nos recuerda, que deberíamos educar o formar a nuestros alumnos para que sean profesionales $y$, por tanto, la enseñanza o los procesos de aprendizaje que hagamos deben estar más centrados en la aplicación práctica del conocimiento y en ser buenos profesionales o unos profesionales competentes, [...]. Hay que dejar de hacer tanto examen o basarnos fundamentalmente en los exámenes para buscar realmente opciones de evaluación y de calificación más ligadas al tema de las competencias. $Y$ claro, esto lo que hace es complicar las cosas. Porque es más complicado evaluar así que evaluar cómo se estaba evaluando tradicionalmente. (GF-2)

Uno de los maestros entrevistados hace referencia a que los sistemas vividos durante la vida escolar y la FIP han influido en su práctica docente actual. Asimismo, ocurre lo mismo con algunos egresados que han participado en el grupo focal, que afirman que los profesores de la FIP que han tenido relación con alumnos de educación primaria conocen mejor los sistemas que deben aplicar durante la formación, para que posteriormente lo apliquen en su práctica docente futura:

To creo que lo que hacen es aplicar esa reproducción de modelos, poner en funcionamiento lo
que han padecido ellos o lo que han sufrido ellos en su etapa escolar: primaria, secundaria o
universidad. Sí hay influencia con lo que han vivido. (ES-3,5)

Mi intuición, y por lo que yo veo, es que el profesor que ha tenido algún tipo de relación en el caso de formación de profesorado con alumnos de primaria, entiende más o menos qué es lo que tiene que hacer con sus alumnos durante la carrera para que luego lo apliquen posteriormente. (GF-5)

\subsection{Objetivo 2: explorar que otros aspectos de la formación inicial o permanente han influido para que el profesorado de educación física de primaria utilice sistemas de EFyC en sus aulas}

Consideramos necesario que para que los futuros maestros hagan partícipes a su alumnado de educación primaria en el proceso de evaluación y de enseñanza-aprendizaje, deben utilizar sistemas de EFyC durante su formación inicial. En el cuadro 5 recogemos los resultados del bloque 2 del cuestionario sobre la utilización de la EFyC.

Cuadro 5. Resultados de los ítems sobre la calificación (escala Likert 1-4)

\begin{tabular}{lcc}
\hline \multicolumn{1}{c}{ ÍTEMS. GRADO DE ACUERDO CON: } & MEDIA & DT \\
\hline $\begin{array}{l}\text { El alumno se autocalifica (parcial o totalmente) } \\
\text { Se califica de forma dialogada y consensuada, entre profesorado y alumnado } \\
\text { (parcial o totalmente) }\end{array}$ & $2,61,916$ \\
$\begin{array}{l}\text { Se califica a partir de la autoevaluación del alumno (parcial o totalmente) } \\
\text { Se califica a partir de la evaluación entre compañeros (coevaluación) (parcial o }\end{array}$ & 2,56 &, 784 \\
totalmente) & 2,44 &, 705 \\
\hline
\end{tabular}

Fuente: Elaboración propia.

Respecto a la participación del alumnado en la evaluación (véase cuadro 4), los datos muestran que los maestros encuestados están bastante de acuerdo con que el alumnado 
participe en los procesos de evaluación y calificación, aunque con pequeñas diferencias entre unos y otros aspectos. Por ejemplo, el mayor grado de acuerdo se da con la realización de procesos de calificación dialogada entre profesor y alumno, mientras que el menor grado de acuerdo se da con que la calificación provenga, parcial o totalmente, de procesos de evaluación entre alumnos.

Dentro de la evaluación, se producen confusiones entre los términos evaluar y calificar. Los maestros entrevistados consideran que la evaluación es un proceso esencial para el aprendizaje, sin embargo, opinan que la calificación es una obligación:

Socialmente la nota es fundamental. (ES-1,5)

Al final tienes que llegar a una nota numérica. (ES-3,5)

Todo termina en una nota por obligación. (ES-2,5)

En este caso, uno de los maestros entrevistados asegura que utiliza una escala graduada para dar el salto de evaluación a calificación, y el alumnado es consciente de la equivalencia de cada grado de consecución desde principio de curso:

El salto de la evaluación a la calificación lo hago a través de escalas graduadas. A través de se ha conseguido o no se ha conseguido según los aspectos que trabajamos, y a partir de ahi el grado de consecución: muy bien, regular, bien... A partir de mis observaciones, del registro que yo tengo, con una escala, pues entonces ver el grado de cumplimiento de los objetivos. (ES$3,5)$

Respecto a la participación del alumnado en su proceso de evaluación, todos los maestros entrevistados coinciden en la importancia del diálogo y el consenso en la evaluación con el alumnado; aunque los instrumentos de evaluación dependen de la unidad didáctica que se esté impartiendo.

Yo normalmente, en la primera unidad didáctica del curso, suelo darles a los alumnos una serie de criterios, requisitos, ítems... que se pueden evaluar a lo largo del curso. Normalmente de una manera democrática. (ES-2,5)

Yo lo que suelo hacer es al final de evaluación hacemos una mini entrevista entre ellos y yo. Dependiendo de las unidades didáctica que hayamos hecho, [..] les damos los instrumentos que hemos recogido, lo hablamos entre ellos y yo, y a partir de ahi establecemos una calificación. Normalmente, al 90\% estamos de acuerdo. (ES-1,5)

En cuanto a la libertad de los maestros para utilizar sistemas de EFyC en sus aulas, nos encontramos con varios puntos de vista. Según uno de los maestros entrevistados asegura que en su centro realizan evaluación formativa, pero que se les exige una calificación, junto con unas observaciones de todas las unidades didácticas que realicen a lo largo del curso. Los otros dos maestros entrevistados sí realizan evaluación formativa, a pesar de las limitaciones que puedan imponer los centros: criterios de calificación fijados, cambios de centros constantes, etc.

To tengo que poner nota por cada unidad didáctica obligatoriamente, porque me obliga el centro en el cuaderno de evaluación. [...] No es coherente que en mi colegio hagamos evaluación formativa y te exijan una nota por unidad... Tenemos que meter un mínimo de observaciones en el cuaderno de evaluación. (ES-1,5)

He usado un año sistemas tradicionales porque tenía un sistema de calidad con unos criterios de calificación fijados por ciclos en primaria, y los sistemas de evaluación y calificación que tenía que llevar a cabo tenía que ser igual al de mis compañeros. [...] Pero me salía de la línea, yo decidía un poco como valoraba otros aspectos: actitudes, valores... (ES-2,5) 
Sigo haciendo evaluación formativa, lo que pasa que los grupos de chavales siempre son diferentes. Empecé haciéndola en educación física en primaria, luego estuve otros 5 años de secundaria y también lo hice y ahora estoy en otro cole de primaria y también lo hago, ahora soy tutor de $1^{\circ}$ de primaria. Vas un poco cambiando, ajustando el sistema a los alumnos que tienes. (ES-3,5)

En cambio, los egresados del grupo focal centran más sus discursos en las limitaciones que establecen las propias universidades para utilizar sistemas de EFyC en FIP: porcentajes mínimos y máximos para examen final, coherencia y grado de cumplimiento de lo que aparece reflejado en las guías docentes, etc.

En nuestra universidad lo que es obligatorio es hacer examen final y lo que es potestativo es hacer continua. $\Upsilon$ puedes hacer las dos cosas. $\Upsilon$ si haces las dos cosas el alumno puede llegar a dos resultados porque ha utilizado los dos procedimientos y se queda con el mejor de los dos. $(\mathrm{GF}-1)$

En nuestro caso, por ejemplo, no puede haber un examen final con más del 40\%. (GF-4)

Entonces, a mí me gustaría ver si realmente las estrategias de evaluación que se plantean en las guías y lo que el profesor hace en el aula. Porque creo que no es así, que al final se evalúa con un examen el $70 \%$ y el 30\% las prácticas. (GF-6)

\section{Discusión y conclusiones}

\subsection{Objetivo 1: examinar en qué medida la evaluación formativa y compartida experimentada durante la FIP ha influido en el tipo de evaluación que realizan actualmente en sus aulas los maestros de educación física de primaria}

Los maestros entrevistados consideran que la FIP recibida ha influido en su práctica docente, pero que no ha sido la suficiente, por lo que han tenido que formarse posteriormente. En cambio, en el grupo focal se aprecian algunas diferencias, en función de la edad de los egresados. Los participantes más jóvenes sí reconocen haber tenido sistemas de EFyC durante su FIP, y que esta experiencia ha influido positivamente en los sistemas de evaluación utilizados en su práctica docente actual. Por otra parte, algunos de los participantes afirman no haber tenido este tipo de experiencias durante su FIP, por lo que se han formado posteriormente de manera autónoma. Estos resultados coinciden, en parte, con las hipótesis señaladas por Palacios y López-Pastor (2013) y Hamodi et al. (2017), en el sentido de que los docentes tienden a realizar la evaluación de manera semejante a como la vivieron ellos mismos como alumnos; pero también muestra lo contrario, que a pesar de no haber tenido experiencias de EFyC durante su FIP, su interés en la temática les lleva a realizar una FPP sobre el tema, para aprender a desarrollar sistemas de EFyC en su práctica docente actual (Barrientos et al., 2018; Molina y López, 2017; Molina et al., 2018).

Por otra parte, los resultados del grupo focal indican que los maestros que han vivido y experimentado sistemas de EFyC durante su FIP consideran que ese tipo de experiencias sí que les ha ayudado a desarrollado la competencia evaluativa, entre otras competencias profesionales y personales. Estos resultados coinciden con otros estudios (Manrique et al., 2011; López-Pastor y Pérez-Pueyo, 2017; Ramírez-García et al., 2018) que afirman que es importante dominar el desarrollo de competencias para el futuro próximo, cuando los alumnos ejerzan como docentes. 
Una de las posibilidades de desarrollar estas competencias es a través de la participación del alumnado en el proceso de evaluación durante la FIP (López-Pastor, 1999, 2009). Los resultados obtenidos en este estudio se pueden contrastar con los encontrados por Ureña et al. (2006), en el que afirman que la autoevaluación puede ayudar a aprender a valorar la calidad de un trabajo.

\subsection{Objetivo 2: explorar que otros aspectos de la formación inicial o permanente han influido para que el profesorado de educación física de primaria utilice sistemas de evaluación formativa y compartida en sus aulas}

La necesidad de desarrollar los sistemas de $\mathrm{EFyC}$ en todos los ámbitos educativos ya está recogido en numerosos estudios (Dochy et al., 2002; Hamodi et al., 2017; Hamodi y LópezPastor, 2012; López-Pastor, 2009; López-Pastor y Pérez-Pueyo, 2017; Palacios y LópezPastor, 2013). En este estudio, los participantes están convencidos de que siempre se pueden implantar sistemas de $\mathrm{EFyC}$ en primaria, a pesar de las limitaciones que un centro te pueda imponer, como, por ejemplo: calificaciones constantes o la utilización del mismo sistema de evaluación que tus compañeros; consideran que siempre hay posibilidades de desarrollar procesos de $\mathrm{EFyC}$ en cualquier materia, aunque sea de forma parcial. Todo ello coincide con los resultados encontrados por numerosos estudios que demuestran que es posible llevar a cabo sistemas de EFyC en todos los ámbitos y niveles educativos (García y López-Pastor, 2015; López-Pastor, 1999, 2009; López-Pastor y Pérez-Pueyo, 2017; López-Pastor et al., 2006).

Los maestros consideran que la participación del alumnado en su proceso de evaluación es beneficiosa. Algunos de los maestros utilizan la calificación dialogada y escalas graduadas para dar el salto de evaluación a calificación y así hacer partícipes a sus alumnos. Respecto a esta idea, investigaciones como la de López-Pastor, González y Barba (2005) y la de López-Pastor y Pérez-Pueyo (2017) también afirman que la implicación del alumnado en su proceso de aprendizaje es conveniente, ya que el alumnado es capaz de utilizar la información que recibe para mejorar su proceso de aprendizaje.

Como conclusión, los resultados muestran que la mayoría de los maestros piensan que sí existe una influencia de las experiencias de $\mathrm{EFyC}$ vividas durante la FIP en su práctica actual en las aulas. Estos resultados parecen indicar que, aunque dicha vivencia se haya dado en pocas asignaturas, ha sido muy importante para conocer una forma de evaluar diferente a la tradicional. Los maestros piensan que la ventaja fundamental de los sistemas de $\mathrm{EFyC}$ es que el alumnado es consciente y mejora su proceso de enseñanza-aprendizaje.

Este estudio puede ser importante para los profesores que ejercen su docencia en la FIP, ya que deben ser conscientes de la importancia de que sus alumnos experimenten sistemas de EFyC durante su formación. También puede ser interesante para maestros de educación primaria de educación física que quieran desarrollar sistemas de $\mathrm{EFyC}$ en sus aulas, por los beneficios que supone.

La principal limitación del estudio es que la muestra de maestros es muy reducida y local, por lo que sería conveniente realizar nuevos estudios con muestras más amplias y en otros contextos. 


\section{Referencias}

Barrientos, E., López-Pastor, V. M. y Molina, M. (2018, octubre). How does the Physical Education Teacher Education influence to carry out Formative Assessment or/and Assessment for Learning Systems? Comunicación presentada en la AIESEP Specialist Seminar 'Future Directions in PE Assessment'. Eindhoven.

Barrientos, E., López-Pastor, V. M. y Pérez-Brunicardi, D. (2019). ¿Por qué hago evaluación formativa y compartida y/o evaluación para el aprendizaje en EF? La influencia de la formación inicial y permanente del Profesorado. Retos, Nuevas tendencias en Educación Física, Deporte y Recreación,36, 37-43.

Buscà, F., Pintor, P., Martínez, L. y Peire, T. (2010). Sistemas y procedimientos de Evaluación Formativa en docencia universitaria: resultados de 34 casos aplicados durante el curso académico 2007-2008. Estudios sobre Educación, 18, 256-276.

Cañadas, L. (2018). La evaluación formativa en la adquisición de competencias docentes en la formación inicial del profesorado de educación fïsica. Tesis doctoral. Universidad Autónoma de Madrid, España.

Castejón, F. J., López-Pastor, V. M., Julián, J. A. y Zaragoza, J. (2011). Evaluación formativa y rendimiento académico en la formación inicial del profesorado de educación física. Revista Internacional de Medicina y Ciencias de la Actividad Física y el Deporte, 11(42), 328-346.

Castejón, F. J., Santos, M. L. y Palacios, A. (2015). Cuestionario sobre metodología y evaluación en formación inicial en educación física. Revista Internacional de Medicina y Ciencias de la Actividad Física y el Deporte, 15(58), 245-267. https://doi.org/10.15366/rimcafd2015.58.004

Córdoba, T., López-Pastor, V. M. y Sebastiani, E. (2018). ¿Por qué Hago Evaluación Formativa en Educación Física? Relato Autobiográfico de un Docente. Estudios Pedagógicos 44(2), 21-38.

Dolchy, F., Segers, M. y Dierick, S. (2002). Nuevas Vías de Aprendizaje y Enseñanza y sus Consecuencias: una Nueva Era de Evaluación. Revista de la Red Estatal de Docencia Universitaria, 2(2), 13-29.

Fraile, A., López-Pastor, V. M., Castejón, J. y Romero, R. (2013). La evaluación formativa en docencia universitaria y el rendimiento académico del alumnado. Aula Abierta 41(2), 23-34.

Fernández, M. (1989). Así enseña nuestra universidad. Salamanca: Hispagraphis.

Flórez, J. (1999). Evaluación de la calidad de la docencia. León: Universidad de León.

García, S. y López-Pastor, V. M. (2015). Evaluación Formativa y Compartida en Educación Infantil. Revisión de una Experiencia Didáctica. Qualitative Research in Education, 4-3, 269298. https://doi.org/10.17583/qre.2015.1269.

Hamodi, C. (2014). La evaluación formativa y compartida en Educación Superior: un estudio de caso (tesis doctoral). Soria: Universidad de Valladolid.

Hamodi, C. y López-Pastor, A. T. (2012). La evaluación formativa y compartida en la Formación Inicial del Profesorado desde la perspectiva del alumnado y de los egresados. Psychology, Society $\Xi^{2}$ Education, 4(1), 103-116.

Hamodi, C., López-Pastor, V. M. y López-Pastor, A. T. (2017). If I experience formative assessment whilst studying at university, will put i tinto practice later as a teacher? Formative and shared assessment in Initial Teacher Education (ITE). Eurepean Journal of Teacher Education, 4O(2), 171-190. https://doi.org/10.1080/02619768.2017.1281909. 
Ibarra, M. S., Rodríguez, G. y Gómez, M. A. (2012). La evaluación entre iguales: beneficios y estrategias para su práctica en la universidad. Revista de Educación, 359, 206-231. https://doi.org/10.4438/1998-592X-RE-2011-359-092.

López-Pastor, V. M. (1999). Prácticas de evaluación en Educación Física: estudio de casos en Primaria, Secundaria y Formación del Profesorado. Valladolid: Universidad de Valladolid.

López-Pastor, V. M. (2008). Desarrollando sistemas de evaluación formativa y compartida en la docencia universitaria. Análisis de resultados de su puesta en práctica en la formación inicial del profesorado. European Journal of Teacher Education, 31(3), 293-311. https://doi.org/10.4995/redu.2009.6232

López-Pastor, V. M. (2009). Evaluación Formativa y Compartida en Educación Superior. Propuestas, técnicas, instrumentos y experiencias. Madrid: Narcea.

López-Pastor, V. M. (2012). Evaluación formativa y compartida en la universidad: clarificación de conceptos y propuestas de intervención desde la Red Interuniversitaria de Evaluación Formativa. Psychology, Society Eं Education, 4(1), 117-130.

López-Pastor, V. M. (2013). Nuevas Perspectivas sobre Evaluación en Educación Física. Revista de Educación Física, 131(29-3), 4-13.

López-Pastor, V. M. y Pérez-Pueyo, A. (2017). Evaluación formativa y compartida en educación: experiencias de éxito en todas las etapas educativas. Buenas prácticas docentes. León: Universidad de León.

López-Pastor, V. M., González, M. y Barba, J. J. (2005) La participación del alumnado en la evaluación: la autoevaluación, la coevaluación y la evaluación compartida. Revista. Tandem. Didáctica de la Educación Física, 17, 21-37.

López-Pastor, V. M., Monjas, R., Gómez, J., Lópezr, E. M., Martín, J.F., González, J. et al. (2006). La Evaluación en Educación Física. Revisión de los modelos tradicionales y planteamiento de una alternativa: la evaluación formativa y compartida. Retos, Nuevas tendencias en Educación Física, Deporte y Recreación, 10, 31-41.

Lorente, E. y Kirk, D. (2013). Alternative democratic assessment in PETE: an action-research study exploring risks, challenges and solutions. Sport, Education and Society, 18, 77-96. https://doi.org/10.1080/13573322.2012.713859

Lorente, E. y Kirk, D. (2016). Student teachers' understanding and application of assessment for learning during a physical education teacher education course. European Physical Education Review, 22(1), 65-81. https://doi.org/10.1177/1356336X15590352

Lorente, E., López-Pastor, V. M. y Kirk, D. (2018). La evaluación participativa en la formación inicial del profesorado. Un caso sobre su utilización en las primeras experiencias profesionales. En E. Lorente-Catalán y D. Martos-García (Eds.), Educación Física y Pedagogía Crítica. Propuestas para la transformación personal y social (pp. 193-213). Lleida: Edicions de la Universitat de Lleida.

Manrique, J. C., López, V. M., Monjas, R., Barba, J. y Gea, J. M. (2011). Implantación de un proyecto de transformación social en Segovia (España): Desarrollo de un programa de deporte escolar en toda la ciudad. Apunts: Educación Física y Deportes, 105, 72-80. https://doi.org/10.5672/apunts.2014-0983.cat.(2011/3).105.07

Martínez-Mínguez, L., Vallés, C. y Romero-Mínguez, M. R. (2015). Estudiantes universitarios: ventajas e inconvenientes de la evaluación formativa. Revista d'Innovació educativa, (14) ,5970. https://doi.org/10.7203/attic. 14.4217 
Mintah, J. K. (2003). Authentic Assessment in Physical Education: Prevalence of Use and Perceived Impact on Students' Self-Concept, Motivation, and Skill Achievement. Measurement in physical education and exercise science, 7(3), 161-174. https://doi.org/10.1207/S15327841MPEE0703_03

Molina, M. y López-Pastor, V. M. (2017). La transferencia de la Evaluación Formativa y Compartida desde la Formación Inicial del Profesorado de Educación Física a la práctica real en Educación Primaria. Revista: Infancia, Educación y Aprendizaje (IEYA), 3(2), 626-631.

Molina, M., López Pastor, V. M., Barrientos, E. y Herranz, M. (2018). Aprendizaje cooperativo y evaluación formativa: ¿por qué llegan a ellos algunos profesores de educación física? En J. Férnandez-Rio, R. Sánchez y A. Méndez, Libro de Actas del XI Congreso Internacional de Actividades Físicas Cooperativas (pp. 115-124). Oviedo: Universidad de Oviedo.

Palacios, A. y López-Pastor, V. M. (2013). Haz lo que yo digo pero no lo que yo hago: sistemas de evaluación del alumnado en la formación inicial del profesorado. Revista de Educación, 361, 279-305. https://doi.org/10.4438/1988-592X-RE-2011-361-143

Porto, M. (1998). La percepción de los estudiantes sobres su evaluación en la universidad. Santiago de Compostela; Universidad de Santiago de Compostela.

Ramírez-García, A., González-Fernández, N. y Salcines-Talledo, I. (2018). Las Competencias Docentes Genéricas en los Grados de Educación. Visión del Profesorado Universitario. Estudios Pedagógicos 44(2), 259-277.

Romero-Martín, R., Castejón-Oliva, F. J. y López-Pastor, V. M. (2015). Divergencias del alumnado y del profesorado universitario sobre las dificultades para aplicar la evaluación formativa. RELIEVE, 21(1). https://doi.org/10.7203/relieve.21.1.5169

Romero-Martín, R., Fraile-Aranda, A., López-Pastor, V. M. y Castejón-Oliva, F. J. (2014). Relación entre sistemas de evaluación formativa, rendimiento académico y carga de trabajo del profesor y del alumno en la docencia universitaria. Infancia y Aprendizaje, 37(2), 310- 341. https://doi.org/10.1080/02103702.2014.918818

Santos, M. A. (1999). 20 paradojas de la evaluación del alumnado en la universidad española. Revista Electrónica Interuniversitaria de Formación del Profesorado, 2(1).

Santos, M., Martínez, L. F. y López-Pastor, V. M. (2009). La Innovación docente en el Espacio Europeo de Educación Superior. Almería: Universidad de Almería.

Silva, I. y López-Pastor, V. M. (2015). ¿Cómo vive el alumnado la evaluación en formación inicial del profesorado? Revista d'innovació educativa. Monográfico: evaluación por competencias en Educación superior 14, 90-100. https://doi.org/10.7203/attic.14.4171

Stake, R. (2005). Investigación con estudio de casos. Madrid: Morata.

Tejedor, F. J. (1998). Las estrategias utilizadas por los profesores universitarios para la evaluación del aprendizaje de los alumnos. Madrid: CIDE.

Trillo, F. y Porto, N. (1999). La percepción de los estudiantes sobre su evaluación en la universidad. Un estudio de caso en la Facultad de Ciencias de la Educación. Revista de Innovación Educativa, 9, 55-75.

Ureña, N., Velandrino, A. P., Alarcón, F. y Ureña, F. (2006). La participación del alumnado en los procesos de evaluación. Una experiencia a partir de la autoevaluación en Educación Física en Educación Primaria. Revista de Educación Física: Renovar la Teoría y Práctica, 103, 11-24. 


\section{Cv de los autores}

\section{Miriam Molina Soria}

Contratada predoctoral de la Facultad de Educación de Segovia (Universidad de Valladolid). Ha publicado diversos artículos científicos. Sus principales líneas de investigación son: Evaluación Formativa y Compartida, y Formación Inicial del Profesorado. ORCID ID: 0000-0003-2974-5535. Email: miriam.molina@uva.es

\section{Víctor M. López Pastor}

Profesor de la Facultad de Educación de Segovia (Universidad de Valladolid). Ha publicado 20 libros y numerosos artículos científicos y profesionales. Sus principales líneas de investigación son: Evaluación Formativa en educación superior, Evaluación Formativa en educación física, Formación del Profesorado, e Investigación-Acción. ORCID ID: o000-0003-2681-9543. Email: vlopez@mpc.uva.es 